九州大学学術情報リポジトリ

Kyushu University Institutional Repository

\title{
Prognostic impact of blood vessel invasion in gastrointestinal stromal tumor of the stomach
}

Yamamoto, Hidetaka

Department of Anatomic Pathology, Graduate School of Medical Sciences, Kyushu University

Kojima, Aya

Department of Anatomic Pathology, Graduate School of Medical Sciences, Kyushu University

Miyasaka, Yoshihiro

Department of Anatomic Pathology, Graduate School of Medical Sciences, Kyushu University

Imamura, Masakazu

Department of Anatomic Pathology, Graduate School of Medical Sciences, Kyushu University

他

http://hdl. handle. net/2324/25997

出版情報: Human Pathology. 41 (10)，pp.1422-1430，2010-10. Elsevier バージョン：

権利関係: (C) 2010 Elsevier Inc. 


\section{Prognostic impact of blood vessel invasion in gastrointestinal stromal tumor}

\section{of the stomach}

Hidetaka Yamamoto, MD, PhD, ${ }^{l}$ Aya Kojima, MD, ${ }^{1}$ Yoshihiro Miyasaka, MD, PhD, ${ }^{1}$ Masakazu Imamura, MD, PhD, ${ }^{1}$ Norimoto Nakamura, MD, PhD, ${ }^{1}$ Takashi Yao, MD, PhD, ${ }^{1,2}$ Masazumi Tsuneyoshi, MD, PhD, ${ }^{1}$ Yoshinao Oda, MD, PhD ${ }^{1}$

1, Department of Anatomic Pathology, Graduate School of Medical Sciences, Kyushu University, Fukuoka, Japan

2, Department of Human Pathology, Graduate School of Medicine, Juntendo University, Tokyo, Japan

\section{Address for correspondence and reprint requests:}

Hidetaka Yamamoto, M.D., Ph.D.

Department of Anatomic Pathology, Graduate School of Medical Sciences, Kyushu University, 3-1-1 Maidashi, Higashi-ku, Fukuoka, 812-8582, Japan

TEL: 81-92-642-6061 FAX: 81- 92-642-5968

e-mail: hidetaka@surgpath.med.kyushu-u.ac.jp

Key Words: gastrointestinal stromal tumor, stomach, metastasis, blood vessel invasion, grade 


\section{Abstract}

Gastrointestinal stromal tumors (GISTs) have a wide spectrum of biologic behavior, and occasional cases show liver metastases. The modified risk grade based on tumor size and mitotic counts has been proposed to predict the biologic behavior in gastric GISTs. Blood vessel invasion (BVI) is important in the development of metastasis of various kinds of cancer. The aim of this study was to elucidate the potential role of BVI in gastric GISTs. BVI was found in 17 of 122 cases (13.9\%) of GISTs, and was significantly correlated with larger tumor size, higher mitotic count and higher modified risk grade. Among 83 cases of primary, localized gastric GISTs available for follow-up information, liver metastasis was observed in 14 cases (16.9\%). When BVI was positive in the primary tumor, liver metastasis occurred in $80 \%$ of cases after the initial surgery, indicating that BVI was a significant risk factor of liver metastasis $(\mathrm{p}<0.0001)$. In univariate and multivariate analyses, tumor size $(>5 \mathrm{~cm})$, mitotic count (>5/50 high-power-fields) and BVI (positive) were significantly associated with a shorter period of disease-free survival. Our results suggest that the evaluation of BVI may be useful for predicting the risk of liver metastasis and aggressive biologic 
Yamamoto et al.- 3 -

behavior of GISTs, and may serve as important information for determining the therapeutic strategies including adjuvant molecular target therapy. 


\section{Introduction}

Gastrointestinal stromal tumor (GIST) is a mesenchymal neoplasm characterized by KIT protein expression and a gain-of-function mutation of the KIT gene or of the platelet-derived growth factor receptor alpha (PDGFRA) gene [1-4]. GISTs have a wide spectrum of biological behavior ranging from benign to malignant. The most frequent sites of metastases are the liver and peritoneum. However, it is difficult to draw a sharp line between benign and malignant lesions based on histological findings alone. Previous studies have reported the prognostic significance of tumor size, mitotic counts, Ki-67 labeling index, KIT mutation subtype, angiogenesis, and aberrant expression of cell-cycle regulators [5-9].

Based on the consensus approach at the National Institutes of Health (NIH) in 2001, Fletcher et al. have recommended to use of risk assessment to predict GIST behavior [10]. They categorized GISTs into four groups: very low, low, intermediate and high risk, based of the combination of tumor size (cut-off; 2, 5, or $10 \mathrm{~cm}$ ) and mitotic counts (cut-off; 5/50 high-power-fields [HPFs]) (Table 1). Subsequent studies have confirmed the prognostic value of this grading in a large series of GISTs [9], and this 
"NIH consensus system" has been applied for both gastric and intestinal GIST. Miettinen and colleagues recently proposed the "modified grading system" for prognostic evaluation, based on the analysis of very large numbers of GISTs, including more than 1700 gastric and 900 small intestinal cases (Table 1) [11-13]. The authors pointed out that gastric GISTs show a lower rate of aggressive behavior than small intestinal (jejunal and ileal) GISTs of similar size and/or mitotic activity. Gastric GISTs with low mitotic activity have a relatively favorable prognosis despite large tumor size; for example, although both group $3 \mathrm{~b}$ (mitoses $\leq 5 / 50 \mathrm{HPF},>10 \mathrm{~cm}$ ) and group $6 \mathrm{~b}$ (mitoses $>5 / 50 \mathrm{HPF},>10 \mathrm{~cm}$ ) had been classified as "high-risk" by a conventional grading system (NIH consensus system), group 3b was found to have significantly better prognosis than group $6 \mathrm{~b}$ among gastric GISTs [13]. However, even the modified grading system incompletely predicts the prognosis of the individual patient with GIST. Particularly in low to moderate grade GISTs, it is challenging to predict which individuals will undergo an adverse clinical course. Therefore, it is necessary to identify additional prognostic markers.

Blood vessel invasion (BVI) or venous invasion is one of the key steps in the spread 
and metastasis of solid tumors. BVI has been suggested to be a significant prognostic indicator for various types of carcinomas of the stomach, colon, uterus, breast and lung [14-18]. As for GIST, the liver is one of the most common sites of metastasis, whereas lymph node metastasis is very rare $[19,20]$, leading us to hypothesize that GIST cells mainly infiltrate into blood vessels rather than lymphatic vessels, and preferentially metastasize to the liver (hematogenic metastasis). In the current study, we aimed to clarify the prognostic significance of BVI in gastric GIST.

\section{Materials and Methods}

\section{Case materials}

We obtained 122 cases of primary GISTs of the stomach from the files of the Department of Anatomic Pathology of Kyushu University between 1986 and 2005. The 115 cases are primary localized gastric tumors, and the remaining 7 cases had gastric tumors with distant metastasis and/or peritoneal dissemination at the initial operation. The diagnosis of all cases of GIST was made based on the histological features and immunohistochemical expression of KIT. 


\section{Histopathological evaluation}

Each GIST was evaluated for clinicopathological and histological features, including tumor size, mitotic count and tumor grade. Tumors were classified into very low, low, moderate and high-grade groups depending on tumor size (cut off; 2, 5 or $10 \mathrm{~cm}$ ) and mitotic count (cut off; 5 per 50 high-power fields [HPFs]), in accordance with the modified grading classification proposed by Miettinen et al. (Table 1). The tumor grading was assigned by two pathologists (HY and AK).

In the vast majority of our cases, the gross specimen was sliced by a series of parallel incisions approximately 5-10 $\mathrm{mm}$ apart, and an entire area of cut-surface across the greatest tumor dimension was submitted for histological examination. In some cases, additional samples were also taken from other part of tumor to microscopically evaluate the margins of excision and extension into adjacent structures and organs, or to verify other changes such as hemorrhage and necrosis. However, in 2 cases, only a limited part of tumor had been sampled for histological examination.

All slides were then carefully searched for BVI by hematoxylin-eosin (HE) stain. 
The number of histological slides (blocks) evaluated ranged from 1 to 25 , with a mean of 7.3 slides per case and a median of 6 slides per case. The mean and median numbers of slides per $1 \mathrm{~cm}$ of the greatest tumor dimension were 1.3 and 1.1, respectively. BVI was defined as the presence of clusters of GIST cells (tumor thrombi) within the vascular spaces, whether within the tumor or at the tumor rim. If suspicious for BVI by HE stain, the corresponding sections were stained with elastica-van Gieson (EVG) stain to confirm the presence of elastic fibers of the vessel wall. BVI was separated from lymphatic vessel invasion according to the presence of smooth muscle and elastic fibers at the vessel wall. In addition, we distinguished BVI from lymphatic vessel invasion by negative immunoreactivity for D2-40, a sensitive marker for lymphatic endothelial cell. The evaluation of BVI was performed by two pathologists ( $\mathrm{HY}$ and $\mathrm{AK}$ ), who were not aware of the clinical characteristics of the patients.

\section{Immunohistochemical staining}

Histological sections ( $4 \mu \mathrm{m}$-thick) of $10 \%$ formalin-fixed, paraffin-embedded 
samples were used for the immunohistochemical examination. The primary antibodies were as follows: c-kit (polyclonal, A4502, dilution; 1/100, Dako, Carpinteria, CA, USA), CD34 (QB-end-10, dilution; 1/50, Novocastra, Newcastle, UK), D2-40 (prediluted, Nichirei, Tokyo, Japan), and Ki-67 (MIB-1, dilution; 1/100, DAKO). The sections were incubated with primary antibodies for one hour at room temperature, followed by the streptavidin-biotin-peroxidase method (Histofine SAB-PO Kit, Nichirei, Tokyo, Japan). The sections were then reacted in a $3,3^{\prime}$-diaminobenzidine peroxytrichloride substrate solution and counterstained with hematoxylin.

\section{Follow-up and statistical analysis}

The correlation between BVI and various clinicopathological parameters was analyzed by the chi-square test, Mann-Whitney U-test, or Spearman's correlation analysis. We analyzed disease-free survival (DFS), for which the end points included any relapse (local recurrence and/or metastasis) of tumor. Univariate analysis of DFS was performed by the Kaplan-Meier method with the log-rank test. For multivariate analysis of DFS, we used the Cox proportional hazards model. A P-value of less than 
0.05 was considered statistically significant.

\section{Results}

\section{Clinicopathological findings}

The 122 patients comprised 60 men and 62 women, ranging in age from 20 to 93 years (median, 64 years). The tumors ranged from 0.4 to $22 \mathrm{~cm}$ in size (median, $5 \mathrm{~cm}$ ). Mitotic counts varied from 0 to 121 per 50 HPFs (median, 3/50 HPFs) and the Ki-67 labeling index varied from 0 to $23.6 \%$ (median, $3.2 \%$ ). According to the modified risk grading system, 45 cases were classified as very low grade, 30 as low grade, 22 as intermediate grade, and 25 as high grade. The case numbers of each group were as follows: group 1 (11 cases), group 2 (34 cases), group 3a (30 cases), group 3b (5 cases), group 4 (0 case), group 5 (17 cases), group 6a (11 cases), and group 6b (14 cases).

\section{Blood vessel invasion}

BVI was observed in 17 of 122 cases (13.9\%). Lymphatic vessel invasion was not detected by immunohistochemical stain for D2-40 (0/122 cases; $0 \%)$. The degree of 
BVI was varied from case to case, but BVI was detected in a few foci rather than everywhere in the individual tumor in most cases. The BVI tended to be present within or at the periphery of the tumor rather than in the adjacent connective tissue close to the tumor invasive front. When BVI was present within the tumor, careful search of BVI was needed to distinguish it from the fascicle or sheet of tumor cells around the blood vessels. On HE sections, the intravascular tumor cluster was well-demarcated by the vessel wall, and the focus of BVI appeared to be a round to oval-shaped, circumscribed structure separated from the surrounding tumor cells (Figure 1). The fascicles of tumor cell around BVI could be abruptly interrupted by the vessel wall. Another helpful finding to detect BVI was that when an artery was not associated with a vein having a hollow vascular space, the vein close to the artery could be filled with tumor thrombi (Figure 1).

The correlation among the presence of BVI and clinicopathological parameters is shown in Table 2. The presence of BVI was significantly correlated with larger tumor size $(\mathrm{p}=0.006)$, higher mitotic counts $(\mathrm{p}=0.0005)$, higher modified risk grade $(\mathrm{p}<0.0001)$, higher Ki-67 labeling index $(\mathrm{p}=0.0023)$, and the presence of liver metastasis at the 
initial operation $(\mathrm{p}=0.0045)$. Liver metastases were present at the initial operation in 4 cases, of which 3 cases $(75 \%)$ had BVI in the primary tumors. The peritoneal metastases were present in 3 cases, but there was no significant correlation between this factor and BVI in the primary tumor $(\mathrm{p}=0.3875)$.

According to the modified risk classification, BVI was identified in $0 / 45$ cases $(0 \%)$ of very low risk GISTs, $3 / 30(10 \%)$ of low risk, 4/22 (18.2\%) of moderate risk, and 10/25 (40\%) of high risk. By group classification, BVI was found in 3/30 (10\%), 4/17 (23.5\%), 3/11 (27.3), and 7/14 (50\%) cases of groups 3a, 5, 6a and 6b, respectively, but was not found in any other groups.

\section{Liver metastasis and blood vessel invasion}

Follow-up information was available in 83 cases with a median follow-up time of 58.4 months. Seven cases which had liver or peritoneal metastases at the initial operation were excluded from follow-up study. Metastases were observed in 16 of 83 cases $(19.3 \%)$, including 10 cases with liver metastases, 2 with peritoneal metastases and 4 with both liver and peritoneal metastases. Liver metastasis was seen in a total of 
14 out of 83 cases $(16.9 \%)$. By modified risk classification, the liver metastasis was present in $0 / 35$ cases $(0 \%)$ of very low grade GISTs, $1 / 16(6.3 \%)$ of low grade, 3/16 (18.8\%) of moderate grade and 10/16 (62.5\%) of high grade. By group classification, liver metastasis was found in 1/16 (6.3\%), 3/12 (25\%), 3/7 (42.9\%), and 7/9 (77.8\%) cases of groups 3a, 5, 6a and 6b, respectively, but was not found in any other groups (Figure 2).

The presence of BVI in the primary site was significantly correlated with the presence of liver metastasis among 83 cases $(\mathrm{p}<0.0001)$ (Table 3). Among the 10 cases with BVI, liver metastasis was present in a total of 8 cases (80\%), including low (1/1 case; $100 \%)$, moderate (2/3 cases; $66.7 \%)$, and high-grade ( $5 / 6$ cases; $83.3 \%)$ tumors (Table 4). Among the BVI-negative 73 cases, liver metastases were rare in very low ( $0 / 35$ cases; $0 \%)$, low ( $0 / 15$ cases; $0 \%)$ and moderate-grade (1/13 cases; $7.7 \%)$ tumors, but were present in half of the high-grade tumors (5/10 cases; 50\%). Alternatively, among the 14 cases with liver metastasis, BVI was found in a total of 8 cases $(57.1 \%)$, including $1 / 1(100 \%), 2 / 3(66.7 \%)$ and 5/10 (50\%) of the cases of low, moderate, and high-grade tumors, respectively. The vast majority of 
metastasis-negative cases were free from BVI (67/69 cases; 97.1\%).

We also checked the possibility that the number of histological blocks might affect the results of detection of BVI. In the vast majority of our cases, more than 0.5-1 blocks per $1 \mathrm{~cm}$ (1 block per 1-2cm) of tumor size were submitted for histological examination. There was no significant difference in block numbers per size between very low, low, moderate and high-grade tumors (mean; 1.5, 1.2, 1.2 and 1.1/cm, respectively), or between BVI-positive and BVI-negative cases (mean; 1.2 vs $1.3 / \mathrm{cm}$ ). However, in 2 cases, only a limited part of tumor had been sampled for histological examination; one case was a $17 \mathrm{~cm}$, moderate-grade, BVI-negative and metastasis-negative GIST with 4 blocks sampled $(0.2$ block/cm), and the other was a $22 \mathrm{~cm}$, high-grade, BVI-negative and metastasis-positive GIST with 5 blocks sampled (0.2 block $/ \mathrm{cm})$. Among the liver metastasis-positive cases except for inadequately sampled ones, the number of blocks per tumor size was not significantly different between BVI-positive and BVI-negative tumors (mean; 1.1 vs $1.3 / \mathrm{cm}$ ).

\section{Prognostic analysis}


The prognostic values of several factors for DFS were analyzed in 83 cases (Table 5

and Figure 3). Cases having distant metastasis and/or peritoneal dissemination at the initial operation were excluded in this analysis. In univariate analysis, tumor size ( $>5$ cm) $[\mathrm{p}=0.0013]$, mitotic count $(>5 / 50 \mathrm{HPF})[\mathrm{p}<0.0001]$, Ki-67 LI $(>5 \%)[\mathrm{p}<0.0001]$, modified risk grade (high grade) $[\mathrm{p}<0.0001]$, and BVI (presence) $[\mathrm{p}<0.0001]$ were each significantly associated with a shorter period of DFS. In multivariate analysis, tumor size $(>5 \mathrm{~cm})[\mathrm{p}=0.0010]$, mitotic count $(>5 / 50 \mathrm{HPF})[\mathrm{p}=0.0018]$ and BVI (presence) $[\mathrm{p}<0.0001]$ were identified as independent worse prognostic factors for DFS.

\section{Discussion}

\section{Conventional vs modified risk classification of GIST}

In the current study, in addition to the modified risk grading system, the presence of BVI was significantly associated with liver metastasis and worse prognosis in gastric

GISTs. BVI was also correlated with several clinicopathologic factors, including tumor size, mitotic counts and modified risk grade.

The pathological criteria for the potential biological behavior of GIST had been 
controversial. Since the consensus workshop held at NIH in 2001, the risk grading system, which does not draw a sharp line between "benign" and "malignant" lesions, has been in use $[9,10]$. Based on a more detailed subclassification of large numbers of GISTs, Miettinen and colleagues proposed the modified grading system in 2005 and 2006 [11-13]. In the earlier NIH grading system (conventional risk grading), large $(>10 \mathrm{~cm})$ gastric GISTs, irrespective of mitotic activity, had been merged into the same category (high-risk) [10]. In contrast, Miettinen et al. found that even large $(>10 \mathrm{~cm})$ gastric GISTs with a low mitotic rate $(<5 / 50 \mathrm{HPFs})$ (group $3 \mathrm{~b}$ ) had only a $10 \%$ risk for metastasis, whereas similar sized GISTs with increased mitoses (>5/50HPF) (group 6b) have a much higher risk of aggressive behavior (>50\%) [13]. The authors also found that group 3a $(5-10 \mathrm{~cm},<5 / 50 \mathrm{HPFs})$ GIST, which previously had been classified as intermediate risk, had a low rate of recurrence $(3 \%)$. In the current series of gastric GISTs, there was a low percentage of liver metastases in groups $3 \mathrm{a}$ and $3 \mathrm{~b}$, providing evidence that these groups of gastric GISTs are less aggressive than previously assumed. We also found that the modified grade was significantly associated with BVI, liver metastasis and disease-free-survival time, suggesting the advantage of the modified 
grading system for predicting the biological behavior and prognosis of GIST.

\section{Prognostic value of blood vessel invasion in GIST}

In general, the invasion of tumor cells into blood vessels is thought to be one of the

critical steps for establishing hematogenic metastasis to the distant organs. BVI is a well-known worse prognostic indicator in many carcinomas, whereas data on this phenomenon in soft tissue sarcomas are relatively limited [21,22]. Few studies have evaluated the clinicopathological importance of BVI in GIST [23]. In the current study, we found that the presence of BVI was significantly correlated with larger tumor size, higher mitotic count and higher risk-grade, higher rate of liver metastasis and worse prognosis (shorter disease-free survival) in gastric GISTs. Among BVI-positive GISTs, $80 \%$ of cases showed liver metastasis. The findings support the idea that BVI plays an important role in the metastases of GIST. The results also provide evidence that the histopathological evaluation of BVI may be useful for predicting aggressive biologic behavior in GISTs.

The frequency of BVI was much lower in group $3 b$ than in group $6 b$, although both 
GIST groups had large tumor size $(>10 \mathrm{~cm})$. This result is consistent with that of the above-mentioned prognostic difference between the two groups, and may explain in part the biological spectrum among large-sized GISTs, which had been lumped together as high-grade tumor in the earlier classification.

According to the previous reports and our results, the recurrence after surgery is only $10-20 \%$ or less in low or moderate-grade GIST, making it difficult to exactly predict the prognosis of individual cases of these categories based on the modified risk grade alone [11,13]. In the current study, although the prevalence of BVI was low percentage among low to moderate-risk gastric GISTs, the occurrence of liver metastasis was much higher in the BVI-positive cases than the BVI-negative cases (low-risk, $100 \%$ vs $0 \%$; moderate-risk, $66.7 \%$ vs $7.7 \%$, respectively). Therefore, our results suggest that BVI may be helpful for identifying GISTs which have metastatic potential among low and moderate risk tumors.

As for the high-risk group of GISTs, the frequency of liver metastasis was $62.5 \%$ by risk grade alone, but rose to $83.3 \%$ by a combination of risk grade and BVI, suggesting that a combination with high risk grade and BVI may be useful to identify "very high" 
risk GIST. However, in contrast to the trend in low to moderate grade GISTs, BVI-negative high-grade GISTs also showed frequent (50\%) liver metastases. One possible explanation for this discrepancy is sampling error. In our study, BVI was present in the limited sections rather than everywhere in the individual tumor, even in large-sized, high grade GIST. Therefore, the inadequate sampling can result in missing the foci of BVI. This seems to be a similar problem in ovarian mucinous tumors, for which it is commonly recommended that the pathologist should submit a minimum of one block per $1-2 \mathrm{~cm}(0.5-1$ block per $1 \mathrm{~cm})$ of the greatest dimension of the tumor. Extensive sampling is important in search for stromal invasion because ovarian mucinous tumors often are histologically heterogenous with benign, borderline (non-invasive) and malignant (invasive) areas variably present within the same tumor [24]. In the vast majority of our series of GISTs, more than $0.5-1$ block per $1 \mathrm{~cm}$ was submitted for histological examination. In 1 of 6 cases of BVI-negative, metastasis-positive GISTs, apparently fewer sections (total 5 blocks for a $22 \mathrm{~cm}$ tumor [0.2 block per $1 \mathrm{~cm}])$ were submitted for paraffin block, suggesting a possibility that the BVI component had escaped microscopic detection due to an inadequate sampling. 
Among liver metastasis-positive GISTs except for this case, there was no significant difference in the number of blocks examined between the BVI-negative and BVI-positive cases.

The false-negative detection of BVI also might be influenced by which part of mass was cut for histological examination. Because BVI was most often present at the periphery of tumor, extensive sampling from the peripheral portion of tumor may be needed in addition to the sampling from the slice across the greatest tumor dimension.

Another possibility is that tumor cells permeate in the blood vessels which are too small to be detected by light microscopic examination. Several studies of BVI in carcinomas have recommended the use of immunohistochemical staining for endothelial markers such as factor VIII-related antigen, CD31 and CD34 to improve the detection of tumor-infiltrating vessels $[18,25]$. As for GIST, CD34 is not useful for this purpose because many GIST cells are positive for CD34. In our previous study, various degrees of angiogenesis were observed in GISTs by immunohistochemical staining for CD31 [8]. We reviewed the GIST sections previously stained for CD31, but failed to find the definite invasion to small vessels which were undetectable by EVG stain. 
However, we cannot draw a conclusion about the prevalence and clinicopathological importance of small vessel invasion in GIST by this preliminary observation, and thus further study would be expected. We believe that at this time, it is practical to carefully search for the characteristic morphological features of BVI (see Results) on HE section, and, if needed, add special stains such as EVG stain, which seems to be readily available in most pathological laboratories. The method used in this study still showed a significant prognostic value of BVI in GIST.

\section{Potential clinical significance of blood vessel invasion}

Although the mainstay of treatment for localized, primary GIST has been surgical resection, surgical resection alone has been inadequate, with up to $50 \%$ of patients developing recurrence, metastasis or death by tumor during 5 years after resection [26]. Imatinib mesylate (Gleevec $\AA$, Novartis, Basel, Switzerland) is a selective molecular inhibitor of KIT and PDGFRA protein, and has been widely used for the treatment of metastatic GIST. Approximately $80 \%$ of patients with metastatic GIST show at least some clinical response (partial response or stable disease) to imatinib [27-30]. 
Unfortunately, most patients who initially respond to imatinib become resistant to it, and tumor progression eventually occurs, most often after 18-24 months [28,30]. The secondary KIT mutation in the kinase domain is one of the mechanisms of acquired resistance [31]. Therefore, it seems to be important to prevent or delay the recurrence before apparent metastatic tumor formation develops. A recent phase III study has revealed that the postoperative adjuvant chemotherapy with imatinib mesylate significantly improved the recurrence-free survival of patients who underwent surgical resection of primary GIST [32]. The current study showed that the presence of BVI significantly increased the risk of liver metastasis of GIST. These data suggest that the adjuvant imatinib therapy might benefit patients with BVI-positive GIST. Further clinical investigation is needed to confirm this hypothesis.

\section{Conclusion}

In conclusion, we found that BVI was significantly correlated with both liver metastasis and worse prognosis in gastric GIST. BVI may play an important role in the metastasis of GIST. In addition, the evaluation of BVI at the routine pathological 
diagnosis of primary tumor may be useful for predicting the aggressive biologic behavior of GIST, and may serve as important information for determining the therapeutic strategies, including the adjuvant imatinib therapy. 


\section{References}

1. Lasota J, Miettinen M. KIT and PDGFRA mutations in gastrointestinal stromal tumors (GISTs). Semin Diagn Pathol 2006;23:91-102.

2. Hirota S, Isozaki K, Moriyama Y, et al. Gain-of-function mutations of c-kit in human gastrointestinal stromal tumors. Science 1998;279:577-80.

3. Heinrich MC, Corless CL, Duensing A, et al. PDGFRA activating mutations in gastrointestinal stromal tumors. Science 2003;299:708-10.

4. Yamamoto H, Oda Y, Kawaguchi K, et al. c-kit and PDGFRA mutations in extragastrointestinal stromal tumor (gastrointestinal stromal tumor of the soft tissue). Am J Surg Pathol. 2004;28:479-88.

5. Hasegawa T, Matsuno Y, Shimoda T, et al. Gastrointestinal stromal tumor: consistent CD117 immunostaining for diagnosis, and prognostic classification based on tumor size and MIB-1 grade. Hum Pathol 2002;33:669-76.

6. Antonescu CR, Sommer G, Sarran L, et al. Association of KIT exon 9 mutations with nongastric primary site and aggressive behavior: KIT mutation analysis and clinical correlates of 120 gastrointestinal stromal tumors. Clin Cancer Res 2003;9:3329-37.

7. Martin J, Poveda A, Llombart-Bosch A, et al. Deletions affecting codons 557-558 of the c-KIT gene indicate a poor prognosis in patients with completely resected gastrointestinal stromal tumors: a study by the Spanish Group for Sarcoma Research (GEIS). J Clin Oncol 2005;23:6190-8.

8. Imamura $\mathrm{M}$, Yamamoto $\mathrm{H}$, Nakamura $\mathrm{N}$, et al. Prognostic significance of angiogenesis in gastrointestinal stromal tumor. Mod Pathol 2007;20:529-37.

9. Nakamura N, Yamamoto H, Yao T, et al. Prognostic significance of expressions of 
cell-cycle regulatory proteins in gastrointestinal stromal tumor and the relevance of the risk grade. Hum Pathol 2005;36:828-37.

10. Fletcher CD, Berman JJ, Corless C, et al. Diagnosis of gastrointestinal stromal tumors: A consensus approach. Hum Pathol 2002 33:459-65.

11. Miettinen M, Lasota J. Gastrointestinal stromal tumors: pathology and prognosis at different sites. Semin Diagn Pathol 2006;23:70-83.

12. Miettinen M, Makhlouf H, Sobin LH, Lasota J. Gastrointestinal stromal tumors of the jejunum and ileum: a clinicopathologic, immunohistochemical, and molecular genetic study of 906 cases before imatinib with long-term follow-up. Am J Surg Pathol 2006;30:477-89.

13. Miettinen M, Sobin LH, Lasota J. Gastrointestinal stromal tumors of the stomach: a clinicopathologic, immunohistochemical, and molecular genetic study of 1765 cases with long-term follow-up. Am J Surg Pathol 2005;29:52-68.

14. Bodendorf MO, Haas V, Laberke HG, Blumenstock G, Wex P, Graeter T. Prognostic value and therapeutic consequences of vascular invasion in non-small cell lung carcinoma. Lung Cancer 2009;64:71-8.

15. Lauria R, Perrone F, Carlomagno C, et al. The prognostic value of lymphatic and blood vessel invasion in operable breast cancer. Cancer 1995;76:1772-8.

16. Sakuragi N, Takeda N, Hareyama H, et al. A multivariate analysis of blood vessel and lymph vessel invasion as predictors of ovarian and lymph node metastases in patients with cervical carcinoma. Cancer 2000;88:2578-83.

17. Hyung WJ, Lee JH, Choi SH, Min JS, Noh SH. Prognostic impact of lymphatic and/or blood vessel invasion in patients with node-negative advanced gastric cancer. Ann Surg Oncol 2002;9:562-7. 
18. Liang $\mathrm{P}$, Nakada I, Hong JW, et al. Prognostic significance of immunohistochemically detected blood and lymphatic vessel invasion in colorectal carcinoma: its impact on prognosis. Ann Surg Oncol 2007;14:470-7.

19. Agaimy A, Wünsch PH. Lymph node metastasis in gastrointestinal stromal tumours (GIST) occurs preferentially in young patients < or $=40$ years: an overview based on our case material and the literature. Langenbecks Arch Surg 2009;394:375-81.

20. Tashiro T, Hasegawa T, Omatsu M, Sekine S, Shimoda T, Katai H. Gastrointestinal stromal tumour of the stomach showing lymph node metastases. Histopathology $2005 ; 47: 438-9$.

21. Gustafson P, Akerman M, Alvegård TA, et al. Prognostic information in soft tissue sarcoma using tumour size, vascular invasion and microscopic tumour necrosis-the SIN-system. Eur J Cancer 2003;39:1568-76.

22. Engellau J, Bendahl PO, Persson A, et al. Improved prognostication in soft tissue sarcoma: independent information from vascular invasion, necrosis, growth pattern, and immunostaining using whole-tumor sections and tissue microarrays. Hum Pathol 2005;36:994-1002.

23. Hou YY, Lu SH, Zhou Y, et al. Stage and histological grade of gastrointestinal stromal tumors based on a new approach are strongly associated with clinical behaviors. Mod Pathol 2009;22:556-69.

24. Guerrieri C, Högberg T, Wingren S, Fristedt S, Simonsen E, Boeryd B. Mucinous borderline and malignant tumors of the ovary. A clinicopathologic and DNA ploidy study of 92 cases. Cancer 1994;74:2329-40.

25. Mohammed RA, Martin SG, Gill MS, Green AR, Paish EC, Ellis IO. Improved methods of detection of lymphovascular invasion demonstrate that it is the predominant method of vascular invasion in breast cancer and has important 
clinical consequences. Am J Surg Pathol 2007;31:1825-33.

26. Dematteo RP, Gold JS, Saran L, et al. Tumor mitotic rate, size, and location independently predict recurrence after resection of primary gastrointestinal stromal tumor (GIST). Cancer 2008;112:608-15.

27. Blanke CD, Demetri GD, von Mehren $M$, et al. Long-term results from a randomized phase II trial of standard- versus higher-dose imatinib mesylate for patients with unresectable or metastatic gastrointestinal stromal tumors expressing KIT. J Clin Oncol 2008;26:620-5.

28. Blanke CD, Rankin C, Demetri GD, et al. Phase III randomized, intergroup trial assessing imatinib mesylate at two dose levels in patients with unresectable or metastatic gastrointestinal stromal tumors expressing the kit receptor tyrosine kinase: S0033. J Clin Oncol 2008;26:626-32.

29. Demetri GD, von Mehren M, Blanke CD, et al. Efficacy and safety of imatinib mesylate in advanced gastrointestinal stromal tumors. $N$ Engl J Med 2002;347:472-80.

30. Verweij J, Casali PG, Zalcberg J, et al. Progression-free survival in gastrointestinal stromal tumours with high-dose imatinib: randomised trial. Lancet 2004;364:1127-34.

31. Antonescu CR, Besmer $\mathrm{P}$, Guo $\mathrm{T}$, et al. Acquired resistance to imatinib in gastrointestinal stromal tumor occurs through secondary gene mutation. Clin Cancer Res 2005;11:4182-90.

32. Dematteo RP, Ballman KV, Antonescu CR, et al. Adjuvant imatinib mesylate after resection of localised, primary gastrointestinal stromal tumour: a randomised, double-blind, placebo-controlled trial. Lancet 2009;373:1097-104. 


\section{Figure legends}

Figure 1. The representative histological patterns of blood vessel invasion (BVI) of GIST.

A. The intravascular tumor cluster is well-demarcated by the vessel wall, and the focus of BVI appears to be a round to oval-shaped, circumscribed structure separated from the surrounding tumor cells. The vessel wall is pointed by allow heads, and intravascular tumor cell cluster is indicated by asterisk.

B. The venous wall is highlighted by elastica-van Gieson stain (corresponding to A).

C. The vein is filled with the tumor thrombus (pointed by allow heads). The nearby artery has a hollow vascular space with red blood cells (pointed by allow).

D. The artery and vein are highlighted by elastica-van Gieson stain (corresponding to C).

Figure 2. The frequency of blood vessel invasion (BVI) and liver metastasis in each subgroup (A) and modified risk grade (B) among 83 cases of primary gastric GISTs. Gray bars indicate the frequency of BVI, and black bars show that of liver metastasis. 
Figure 3. Kaplan-Meier analysis for 83 patients with primary gastric GISTs. The modified risk grade (A) and blood vessel invasion (B) are each significantly associated with shorter disease-free survival. 
Figure 1

A
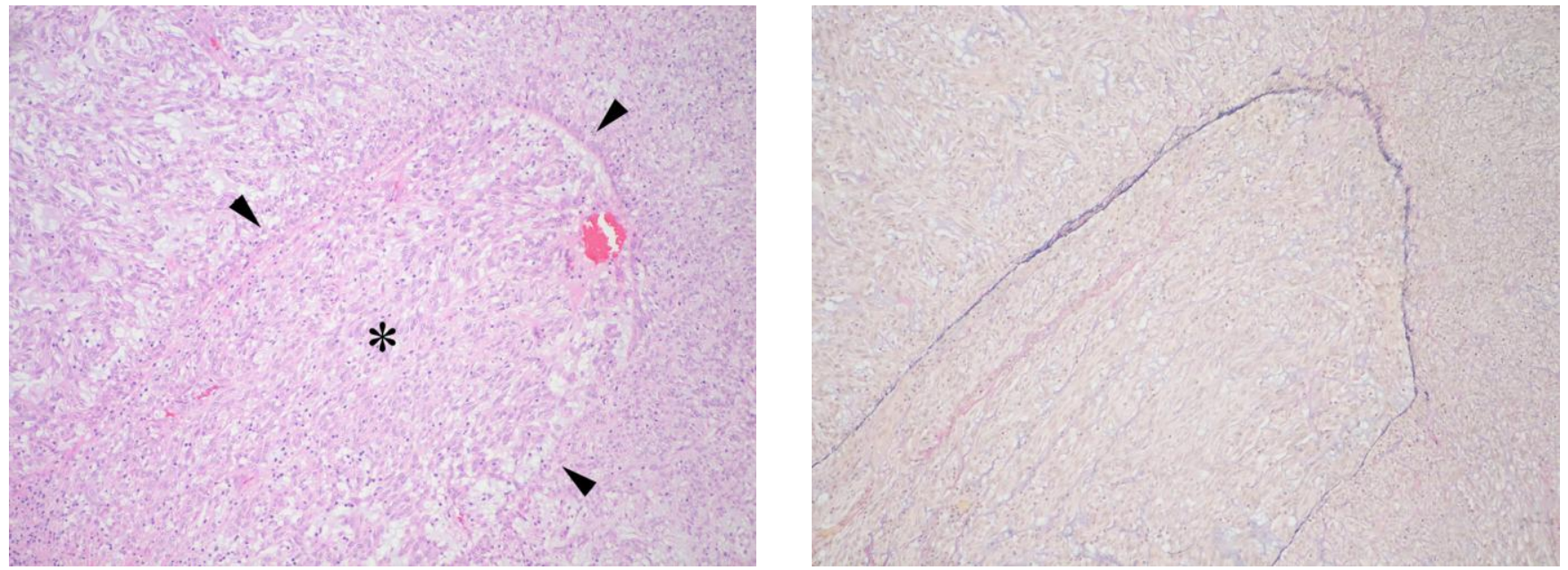

B
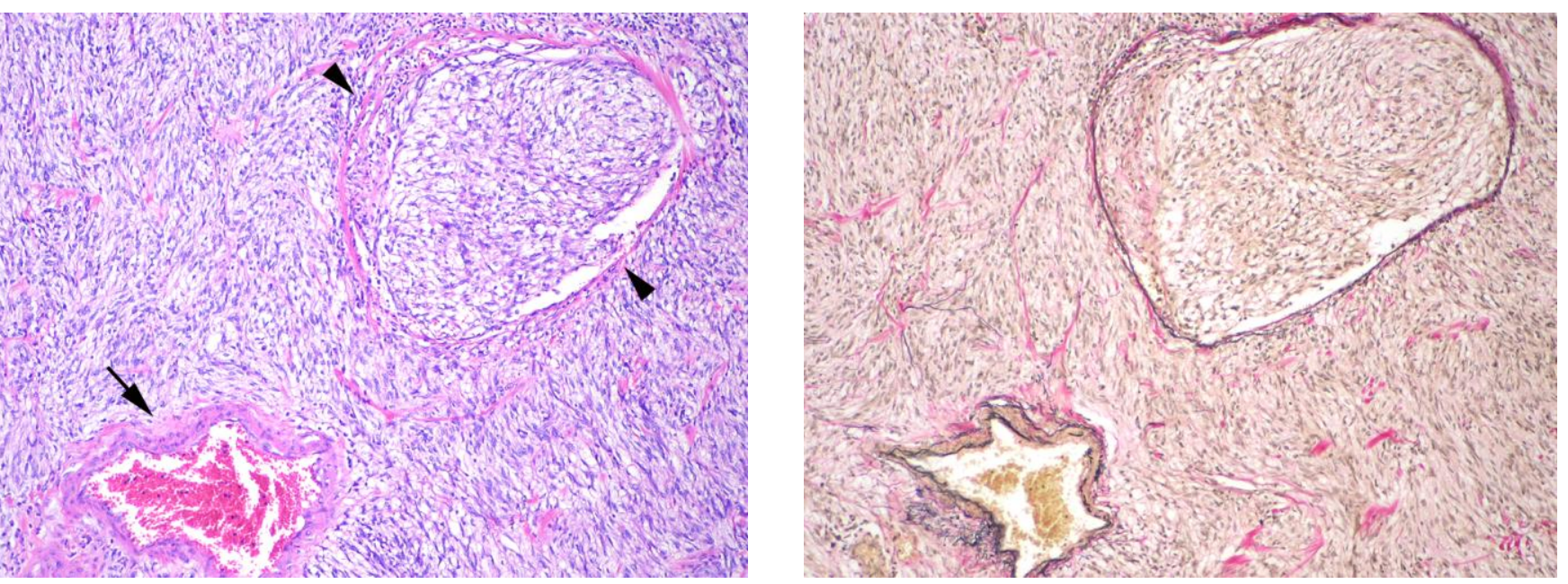
Fig. 2

A

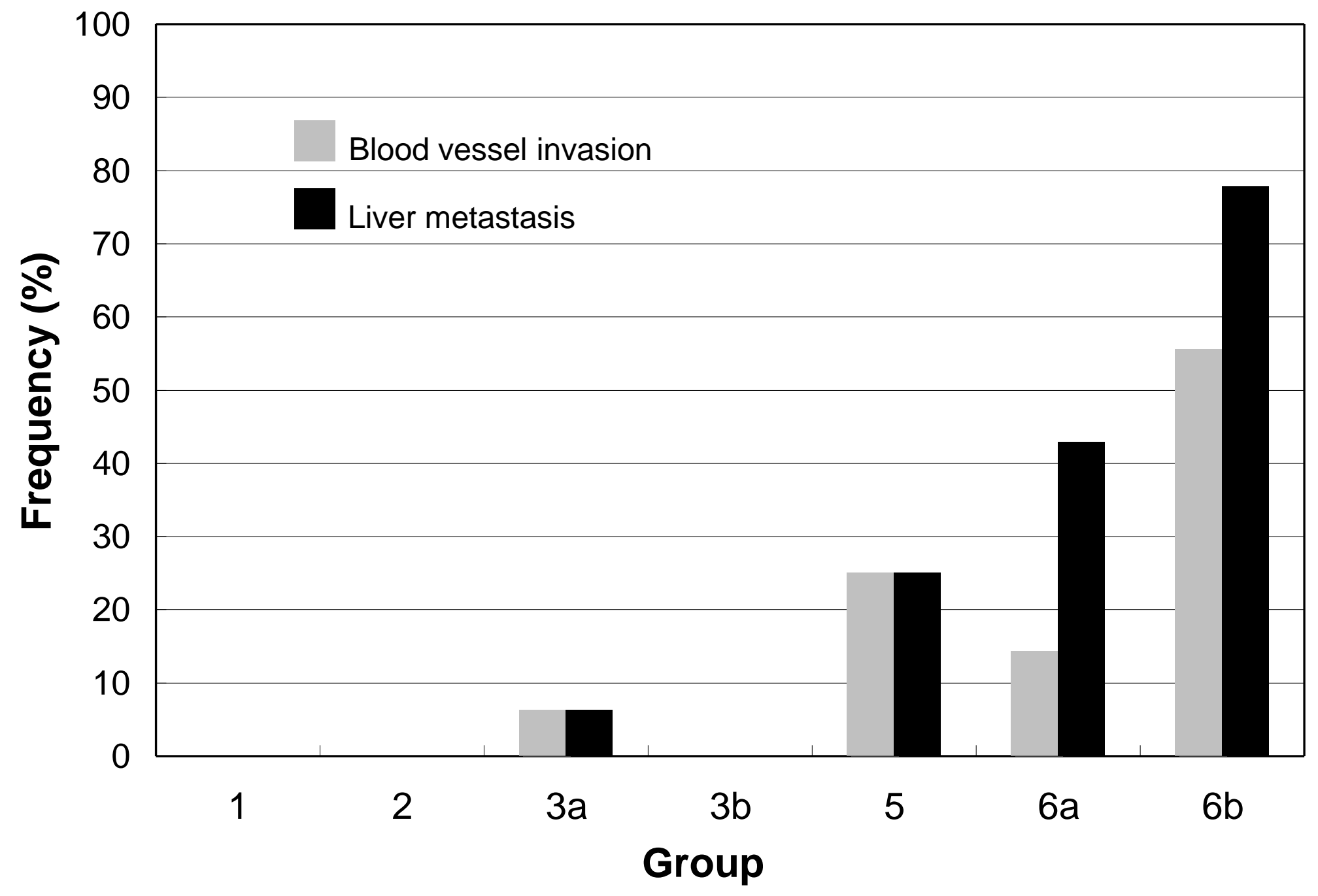


Fig. 2

B

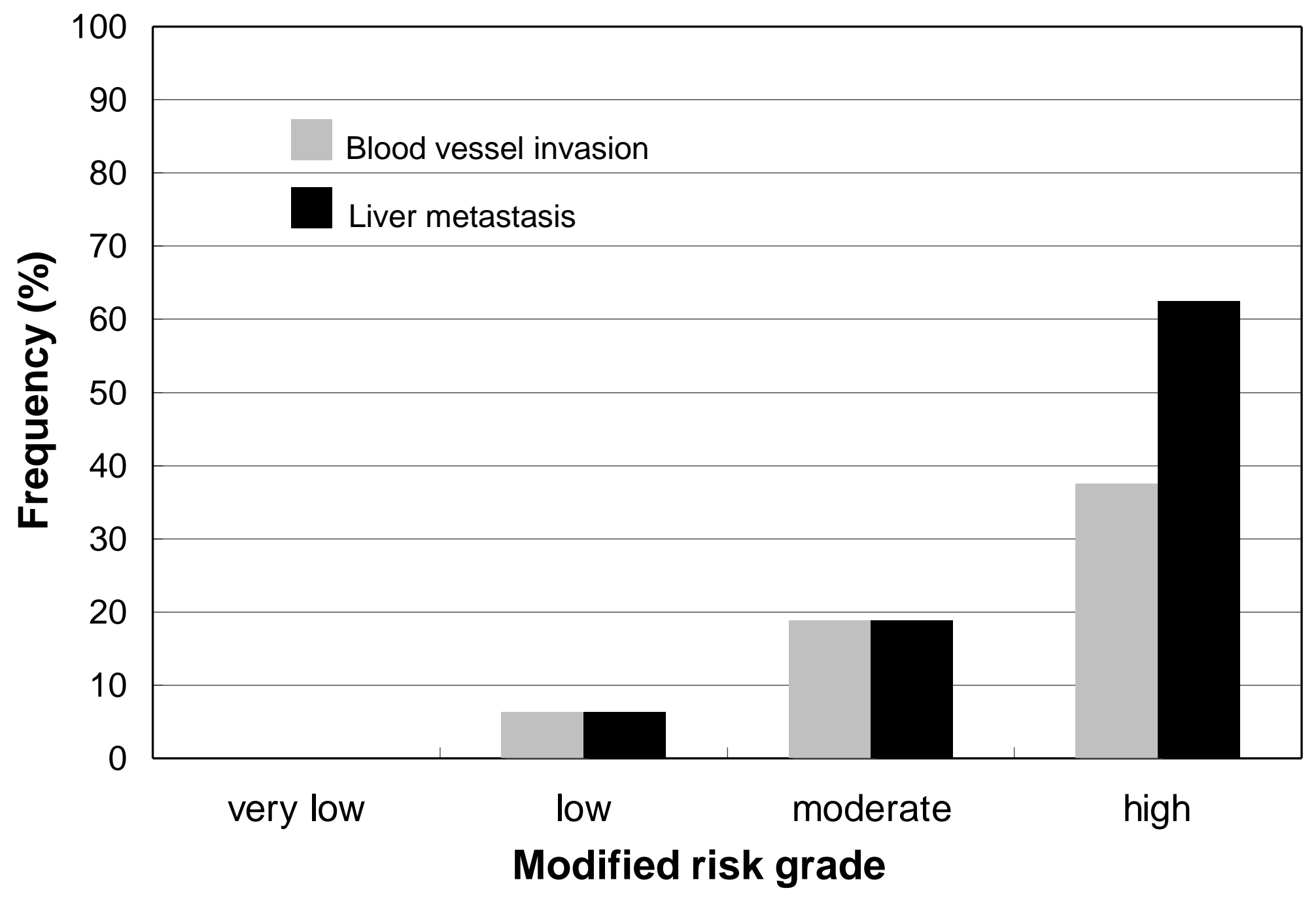




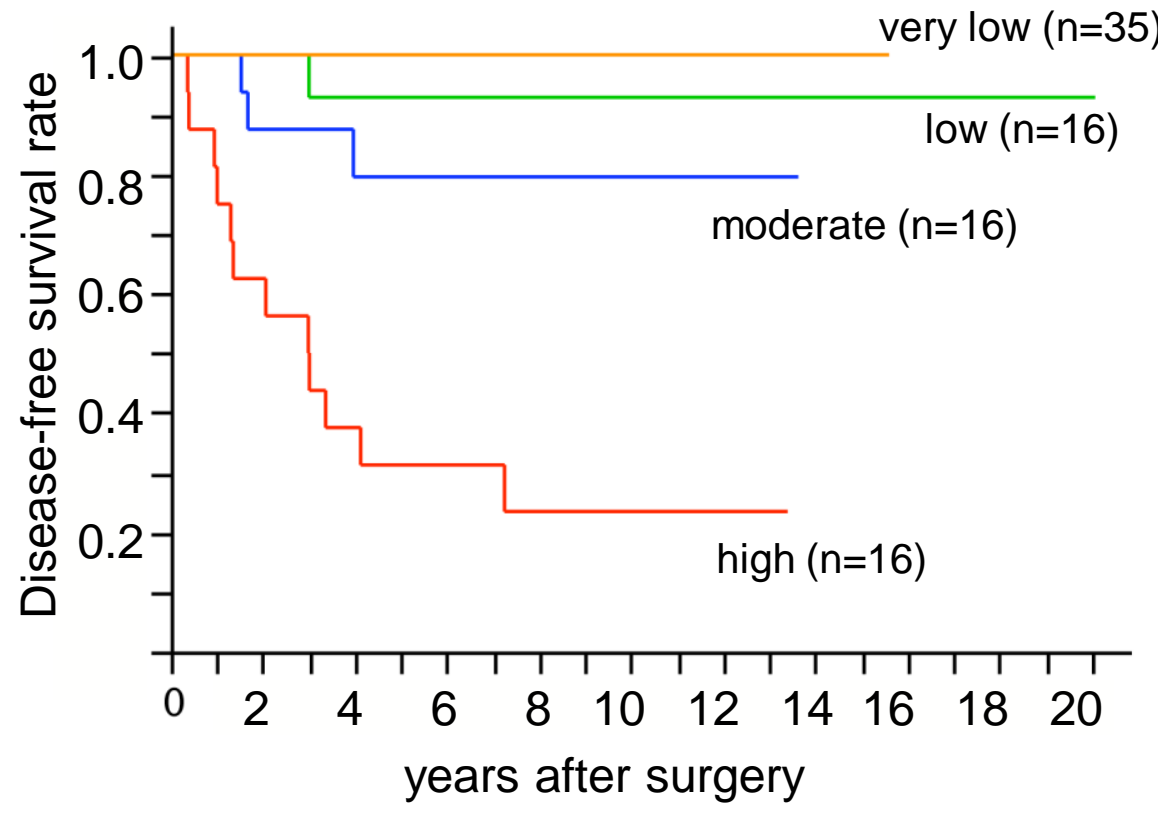

\section{B}

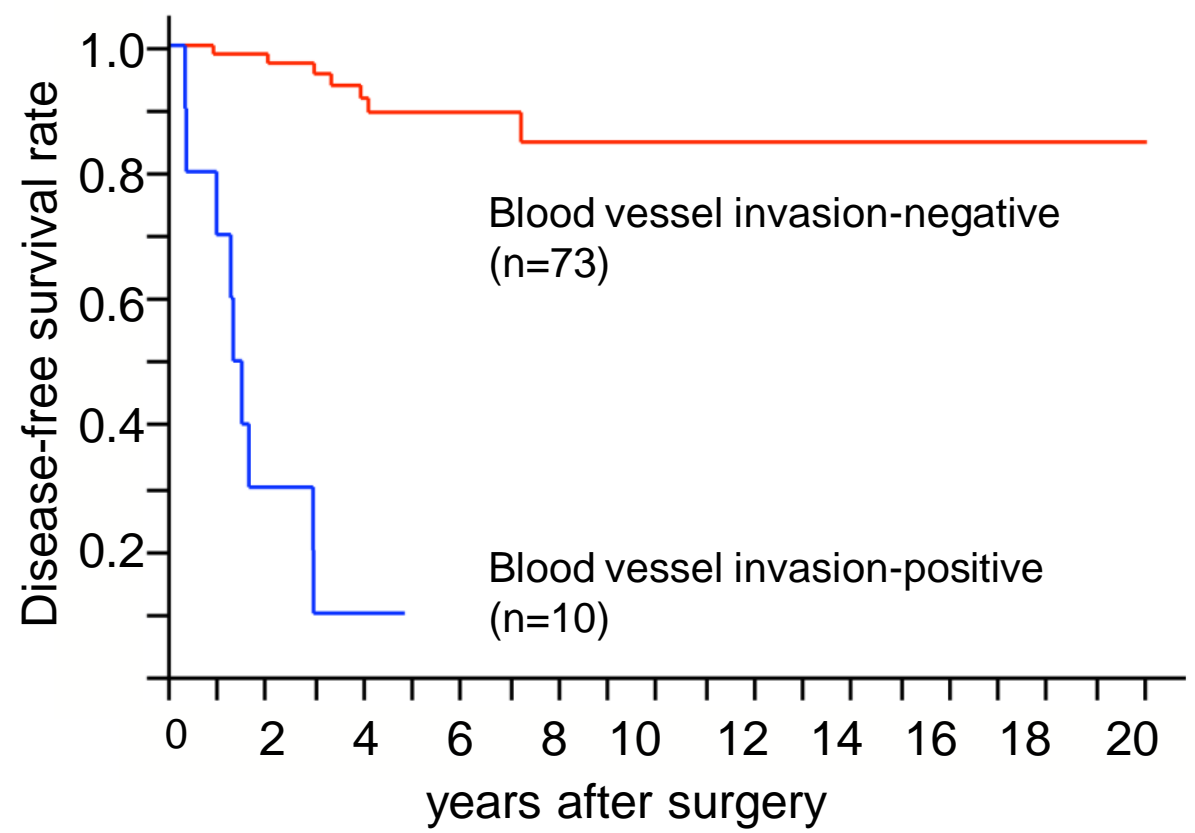


Table 1. The summary of conventional and modified risk grading in gastrointestinal stromal tumor.

\begin{tabular}{|c|c|c|c|c|c|}
\hline \multirow{3}{*}{$\begin{array}{l}\text { Mitoses } \\
\text { (HPF) }\end{array}$} & \multirow{3}{*}{$\begin{array}{l}\text { Size } \\
(\mathrm{cm})\end{array}$} & \multirow[t]{3}{*}{ Group } & \multicolumn{3}{|c|}{ Risk grading system } \\
\hline & & & \multicolumn{2}{|l|}{ Modified $\dagger$} & \multirow{2}{*}{$\begin{array}{l}\text { Conventional }^{\ddagger} \\
\text { Stomach, small intestine }\end{array}$} \\
\hline & & & Stomach & Small intestine & \\
\hline \multirow[t]{4}{*}{$\leq 5 / 50$} & $\leq 2$ & 1 & very low & very low & very low \\
\hline & $>2 \leq 5$ & 2 & very low & low & low \\
\hline & $>5 \leq 10$ & $3 a$ & low & moderate & intermediate \\
\hline & $>10$ & $3 b$ & moderate & high & high \\
\hline \multirow[t]{4}{*}{$>5 / 50$} & $\leq 2$ & 4 & very low* & high* & intermediate \\
\hline & $>2 \leq 5$ & 5 & moderate & high & intermediate \\
\hline & $>5 \leq 10$ & $6 a$ & high & high & high \\
\hline & $>10$ & $6 \mathrm{~b}$ & high & high & high \\
\hline
\end{tabular}

*The modified risk grade for Group 4 tumors is tentative, because the corresponding cases are too few to reliably determine prognosis.

$\dagger$ Reference $11, \$$ Reference 10 
Table 2. The correlation between blood vessel invasion and clinicopathological parameters in 122 cases of gastrointestinal stromal tumors of the stomach.

\begin{tabular}{|c|c|c|c|c|}
\hline & & \multicolumn{3}{|c|}{ Blood vessel invasion } \\
\hline & & positive $(\mathrm{n}=17)$ & negative $(n=105)$ & $p=$ \\
\hline Age* & & $59(48.3-72.3)$ & $66(55-74)$ & 0.2303 \\
\hline \multirow[t]{2}{*}{ Sex } & $M(n=60)$ & 12 & 48 & 0.0541 \\
\hline & $F(n=62)$ & 5 & 57 & \\
\hline Tumor size $(\mathrm{cm})^{*}$ & & $7.9(5.4-14.5)$ & $4.5(3.5-7)$ & 0.006 \\
\hline Mitoses (/50HPF)* & & $16.5(6.8-41)$ & $2(1-6)$ & 0.0005 \\
\hline Ki-67 index $(\%)^{*}$ & & $8.9(4.9-14.8)$ & $2.9(1.2-5)$ & 0.0023 \\
\hline \multicolumn{2}{|c|}{ Liver metastases at the initial operation } & & & 0.0045 \\
\hline & positive $(n=4)$ & 3 & 1 & \\
\hline & negative $(\mathrm{n}=118)$ & 14 & 104 & \\
\hline \multicolumn{2}{|c|}{ Peritoneal metastases at the initial operation } & & & 0.3875 \\
\hline & positive $(n=3)$ & 1 & 2 & \\
\hline & negative $(\mathrm{n}=119)$ & 16 & 103 & \\
\hline \multirow[t]{9}{*}{ Group } & & & & - \\
\hline & $1(\mathrm{n}=11)$ & $0(0 \%)$ & 11 & \\
\hline & $2(n=34)$ & $0(0 \%)$ & 34 & \\
\hline & $3 a(n=30)$ & $3(10 \%)$ & 27 & \\
\hline & $3 b(n=5)$ & $0(0 \%)$ & 5 & \\
\hline & $4(n=0)$ & - & - & \\
\hline & $5(n=17)$ & $4(23.5 \%)$ & 13 & \\
\hline & $6 \mathrm{a}(\mathrm{n}=11)$ & $3(27.3 \%)$ & 8 & \\
\hline & $6 \mathrm{~b}(\mathrm{n}=14)$ & $7(50 \%)$ & 7 & \\
\hline \multirow[t]{5}{*}{ Modified risk grade } & & & & $<0.0001$ \\
\hline & very low $(n=45)$ & $0(0 \%)$ & 45 & \\
\hline & low $(n=30)$ & $3(10 \%)$ & 27 & \\
\hline & moderate $(n=22)$ & $4(18.2 \%)$ & 18 & \\
\hline & $\operatorname{high}(\mathrm{n}=25)$ & $10(40 \%)$ & 15 & \\
\hline
\end{tabular}

* Values were expressed as median levels and interquartile range in parenthesis 
Table 3. The correlation between blood vessel invasion and liver metastases in 83 cases of gastrointestinal stromal tumors of the stomach.

\begin{tabular}{lllll}
\hline & & \multicolumn{3}{l}{ Liver metastasis } \\
positive & negative & \\
$(\mathrm{n}=14)$ & $(\mathrm{n}=69)$ & $\mathrm{p}<0.0001$ \\
\hline Blood vessel invasion & $\begin{array}{l}\text { positive } \\
(\mathrm{n}=10)\end{array}$ & 8 & 2 & \\
$\begin{array}{l}\text { negative } \\
(\mathrm{n}=73)\end{array}$ & 6 & 67 & \\
\hline
\end{tabular}


Table 4. The correlation among blood vessel invasion, liver metastasis and modified risk grade in 83 cases of primary gastrointestinal stromal tumors of the stomach.

\begin{tabular}{llllll}
\hline & very low & low & moderate & high & total \\
& $(\mathrm{n}=35)$ & $(\mathrm{n}=16)$ & $(\mathrm{n}=16)$ & $(\mathrm{n}=16)$ & $(\mathrm{n}=83)$ \\
\hline Blood vessel invasion & $0 / 35$ & $1 / 16$ & $3 / 16$ & $6 / 16$ & $10 / 83$ \\
& $(0 \%)$ & $(6.3 \%)$ & $(18.8 \%)$ & $(37.5 \%)$ & $(12.5 \%)$ \\
Liver metastases & $0 / 35$ & $1 / 16$ & $3 / 16$ & $10 / 16$ & $14 / 83$ \\
& $(0 \%)$ & $(6.3 \%)$ & $(18.8 \%)$ & $(62.5 \%)$ & $(16.9 \%)$ \\
Liver metastases among BVI-positive tumors & $0 / 0$ & $1 / 1$ & $2 / 3$ & $5 / 6$ & $8 / 10$ \\
& - & $(100 \%)$ & $(66.7 \%)$ & $(83.3 \%)$ & $(80 \%)$ \\
Liver metastases among BVI-negative tumors & $0 / 35$ & $0 / 15$ & $1 / 13$ & $5 / 10$ & $6 / 73$ \\
& $(0 \%)$ & $(0 \%)$ & $(7.7 \%)$ & $(50 \%)$ & $(8.2 \%)$ \\
BVI among liver metastases-positive tumors & $0 / 0$ & $1 / 1$ & $2 / 3$ & $5 / 10$ & $8 / 14$ \\
& - & $(100 \%)$ & $(66.7 \%)$ & $(50 \%)$ & $(57.1 \%)$ \\
BVI among liver metastases-negative tumors & $0 / 35$ & $0 / 15$ & $1 / 13$ & $1 / 6$ & $2 / 69$ \\
& $(0 \%)$ & $(0 \%)$ & $(7.7 \%)$ & $(16.7 \%)$ & $(2.9 \%)$ \\
\hline
\end{tabular}

BVI, blood vessel invasion 
Table 5. The univariate and multivariate analysis of prognostic factors in 83 cases of primary gastrointestinal stromal tumors of the stomach.

\begin{tabular}{lll}
\hline & $\begin{array}{l}\text { Disease-free survival } \\
\text { Univariate }(p=)\end{array}$ & Multivariate $(p=)$ \\
\hline Tumor size $(>5 \mathrm{~cm})$ & 0.0013 & 0.001 \\
Mitoses $(>5 / 50 \mathrm{HPF})$ & $<0.0001$ & 0.0018 \\
Ki-67 index $(>5 \%)$ & $<0.0001$ & 0.2076 \\
Modified risk grade (high) & $<0.0001$ & N.E. \\
Blood vessel invasion (positive) & $<0.0001$ & $<0.0001$ \\
\hline
\end{tabular}

N.E., not evaluated 